\title{
Inter-Group Variation in Non-Conceptive Sexual Activity in Female Japanese Macaques: Could it be Cultural?
}

\author{
Jean-Baptiste Leca ${ }^{1 *}$, Noëlle Gunst ${ }^{1}$, Lydia Ottenheimer Carrier $^{1}$, and Paul L. Vasey ${ }^{1}$ \\ ${ }^{1}$ University of Lethbridge \\ *Corresponding author (Email: jbtleca@yahoo.com)
}

Citation - Leca, J-B., Gunst, N., Carrier, L. O., \& Vasey, P. L. (2014). Inter-group variation in non-conceptive sexual activity in female Japanese macaques: Could it be cultural? Animal Behavior and Cognition, 1(3), 387-409. doi: 10.12966/abc.08.12.2014

\begin{abstract}
We compared two non-conceptive sexual behavioral patterns (female-male mounting - FMM - and female-female mounting - FFM) across four free-ranging groups of Japanese macaques (Macaca fuscata) living at three different field sites in Japan (Arashiyama, Minoo, and Jigokudani). We found marked inter-group differences and covariation in the frequency and form of FMM and FFM. This result supports the view that FMM and FFM in Japanese macaques are developmentally and evolutionarily linked. The customary occurrence, high prevalence, and great diversity of FMM and FFM at Arashiyama may be the result of combined favorable socio-demographic conditions, namely few resident males, most of them being old, sexually under-motivated, and less aggressive and controlling than the average male Japanese macaques. We suggest that FMM and FFM may be cultural sexual practices in the Arashiyama-E group. In most other populations, all the aforementioned favorable sociodemographic conditions are not met, and although female mounting may occasionally be expressed by several group members, it does not reach the group-level tradition status. Our cultural interpretation of female mounting in Japanese macaques is consistent with evidence of the social transmission of courtship behaviors and mating preferences in various animal taxa, including nonhuman primates and humans. Our study may have implications for the evolution of non-conceptive sexuality in humans, including sexual fluidity in women.
\end{abstract}

Keywords - Female-male mounting, Female-female mounting, Male senescence, Sex ratio, Sexual coercion, Sexual harassment, Cultural zone, Primates.

Traditionally, it has been thought that substantial intraspecific variation in sexual behavior does not occur. One of the most basic tenets of evolutionary biology is that unambiguous, thus invariant, mating signals should be selected in order to ensure reproductive isolation among species (Dobzhansky, 1937). Indeed, courtship behavior provides a textbook case of what early ethologists called "fixed action patterns" (Lorenz, 1970). However, there is growing evidence for geographic variation in mating behavioral patterns among conspecific populations and across various animal taxa, including insects, fish, birds, and mammals (Verrell, 1999). Such differences are generally attributed to genetic, ecological, socio-demographic, or cultural factors, or a combination of these causes (Verrell, 1999).

The study of non-conceptive sexual patterns, such as female mounting, is particularly useful for researchers interested in understanding inter-group behavioral variation within a given species. Whereas male mounting posture should be optimal (i.e., precisely coordinated and invariant) in order to achieve penile intromission during heterosexual copulation (e.g., Gunst, Leca, \& Vasey, 2013), female mounting is less functionally constrained, which allows for more flexible and arbitrary behavioral patterns (Stephenson, 1973). Indeed, analysis of male and female mounting postures and pelvic movement in Japanese macaques (Macaca fuscata) is consistent with this claim (Vasey, Foroud, Duckworth, \& Kovacovsky, 2006). According to Stephenson (1973), behavioral arbitrariness is a function of individual 
experience, which can be influenced culturally through social interactions with other group members. Arguably, the apparent lack of direct fitness consequences and the arbitrariness of female mounting behavior make it easier to rule out obvious ecological factors and thereby examine cultural factors as potential causes of intraspecific variation ( $c f$. Leca, Gunst, \& Huffman, 2007).

Female mounting has been reported in a variety of mammal species (Beach, 1968; Dagg, 1984). When directed to males, it is referred to as female-male mounting (hereafter FMM). When directed to females, it is referred to as female-female mounting (hereafter FFM). Neither form of mounting directly results in conception. However, female mounting is not necessarily devoid of functional aspects. FMM is often considered a proceptive sexual behavior, i.e., a form of super-solicitation directed to males that may prompt subsequent male-female copulations (Beach, 1968; Dagg, 1984). Like most same-sex sexual behaviors, FFM is often viewed as socio-sexual, that is sexual in its structure but performed for its adaptive social functions (Wickler, 1967). Addressing the emergence, expression and maintenance of intraspecific variation in female mounting may further our understanding of how natural selection results in the evolution of various behavioral patterns, including those that seem to lack obvious reproductive or survival benefits.

With regard to intraspecific variation in non-conceptive sexuality, the Japanese macaque is an ideal primate species model for several reasons. In addition to engaging in reproductive sex (e.g., Pavelka \& Fedigan, 1999), certain populations exhibit various forms of homosexual and heterosexual nonconceptive sex, including FMM (Gouzoules \& Goy, 1983; O’Neill, Fedigan, \& Ziegler, 2006; Vasey \& Duckworth, 2008) and FFM (Vasey, 2002, 2004; Vasey \& Duckworth, 2006). A pioneering study of Japanese macaques revealed that FMM was present in two groups and absent in another group (Stephenson, 1973). Several lines of evidence suggest that the frequency and prevalence of FFM (i.e., number of FFM relative to observation time, and percentage of female group members performing FMM, respectively) vary considerably both between geographically isolated groups and within groups over time, raising the possibility that intraspecific variation in this behavioral pattern is influenced by genetic components (e.g., population-specific haplotypes), demographic variables (e.g., socionomic sex ratio), and/or cultural factors (Nakagawa, Nakamichi, \& Yamada, 2011; Vasey \& Gauthier, 2000; Vasey \& Jiskoot, 2010; Wolfe, 1986).

In terms of development, structure, and physiology, FMM and FFM in Japanese macaques bear striking resemblances, as both are characterized by similar mounting postures and sex hormone fluctuations, which occur during temporary, but exclusive, sexual relationships (i.e., consortships; O'Neill et al., 2006; Vasey, 2007; Vasey \& Duckworth, 2006, 2008; Vasey \& VanderLaan, 2012). Vasey (2007) has suggested that FMM and FFM are evolutionarily linked, with the former being an adaptation and the latter being a by-product of that adaptation.

However, systematic inter-group comparison of both FMM and FFM, and their possible covariation, in Japanese macaques is lacking. Furthermore, the socio-demographic and cultural factors that may influence the expression, frequency, and maintenance of these two types of female mounting in this species remain to be more fully explored. Japanese macaques exhibit substantial inter-group differences in their socionomic sex ratio, dominance style, and levels of agonism and affiliation (Nakagawa, 2010; Zhang \& Watanabe, in press), thereby facilitating tests of socio-demographic hypotheses for intraspecific variation in female mounting. Finally, Japanese macaques are renowned for their group-specific behavioral innovations and traditions in various domains, including tool use (Leca, Gunst, \& Huffman, 2010), foraging techniques (Itani \& Nishimura, 1973), communication patterns (Green, 1975; Stephenson, 1973), grooming styles (Tanaka, 1998), and playing activities (Leca et al., 2007). This species has demonstrated the capacity for cultural transmission of arbitrary behavioral patterns (Leca et al., 2007; Sakura, 1989; Stephenson, 1973). Taken together, these features make the Japanese macaque an obvious candidate for the study of the socio-cultural conditions underlying intraspecific variation in female mounting.

In this study, we compared two non-conceptive sexual behavioral patterns (namely, FMM and FFM) across four free-ranging groups of Japanese macaques living at three different field sites in Japan. To explain intra-group similarities and inter-group differences in the frequency, prevalence, form, and 
context of expression of FMM and FFM, we devised a series of predictions generated from five nonmutually exclusive hypotheses (Table 1). Our first objective was to provide further evidence that FMM and FFM in Japanese macaques are developmentally and evolutionarily linked (Leca, Gunst, \& Vasey, in review; Vasey, 2007). Therefore, we tested the "female mounting covariation" hypothesis, which holds that the frequency and form of FMM and FFM covary across groups.

Our second objective was to investigate three possible socio-demographic origins of inter-group differences in the frequency of FMM. First, previous research in various mammal taxa showed that (1) FMM could function as a "super-solicitation" and serve to stimulate males with low levels of sexual motivation and performance (Beach, 1968; Dagg, 1984), and (2) senescent males were less sexually motivated and efficient than younger males (Sitzmann, Urbansky, \& Ottinger, 2008). Therefore, we tested the "senescent male mates" hypothesis, which holds that group-specific expression of FMM is related to the percentage of senescent (thus sexually under-motivated) males in the group. Second, previous research in various mammal taxa showed that: (1) FMM could serve to focus the male's attention and prevent him from moving away, in the context of high female-female competition for male mates (Beach, 1968; Dagg, 1984), and (2) female-female competition for male mates is higher in groups with femalebiased sex ratios (Kvarnemo \& Ahnesjö, 2002). Therefore, we tested the "few male mates" hypothesis, which holds that group-specific expression of FMM is related to female intrasexual competition, which is higher in groups with more female-biased sex ratios. Third, previous research suggested that (1) intergroup differences in the level of male aggressiveness might affect sexual behavior and reproductive success in primates (Sapolsky \& Share, 2004, Silk, 2007), and (2) inter-group differences in the level of male aggressiveness in Japanese macaques have been reported (Nakagawa, 2010; Zhang \& Watanabe, in press). Therefore, we tested the "mellow male mates" hypothesis, which holds that FMM is more tolerated in groups with less coercive, less controlling, and more affiliative male mates.

Our third objective was to address inter-group differences in the expression and maintenance of FFM. Previous research showed that sexual harassment - defined as a third-party individual interrupting a consortship by displacing or aggressing both consort partners - was a male-typical strategy for disrupting female homosexual consortships in Japanese macaques (Vasey, 2004). Therefore, we tested the "mellow third-party males" hypothesis, which holds that FFM is more tolerated in groups with lower levels of sexrelated aggressiveness by third-party males (such as sexual harassment). We argued that inter-group differences in the expression and maintenance of FMM and FFM could be attributed to local sociodemographic conditions and cultural factors.

\section{Method}

\section{Study Species and Four Main Study Groups}

Japanese macaques are seasonal breeders. Females ovulate only during the breeding season (i.e., autumn and winter months). Males are mount-series ejaculators, with most copulations occurring within series of mount-dismount sequences and the male ejaculating at the end of the last mount. Like other macaque species, they live in mixed-sex groups and are characterized by a promiscuous mating system (Dixson, 2012).

Observations were conducted on four free-ranging provisioned groups of Japanese macaques living at three sites across Japan: Arashiyama-E group (Kyoto Prefecture), Minoo-F and Minoo-L groups (Osaka Prefecture), and Jigokudani-A1 group (Nagano Prefecture; Figure 1). Minoo-F and Minoo-L were neighboring groups because their respective home ranges overlapped and inter-group encounters occurred. All the study subjects were well habituated to human presence. Group sizes and compositions are provided in Table 2. 


\begin{tabular}{|c|c|c|}
\hline Hypotheses & Predictions & $\begin{array}{c}\text { Supported } \\
\text { (see Results) }\end{array}$ \\
\hline \multirow[t]{4}{*}{ 1: Female mounting covariation } & 1a: Frequency of FMM and FFM should be positively correlated across groups & Yes \\
\hline & 1b: Prevalence of FMM and FFM should be positively correlated across groups & Partly \\
\hline & 1c. Within groups, most females performing FMM should also perform FFM, and vice versa & No \\
\hline & 1d: Structural diversity of FMM and FFM should be positively correlated across groups & Yes \\
\hline \multirow[t]{3}{*}{ 2: Senescent male mates } & $\begin{array}{l}\text { 2a: During heterosexual consortships, females should be more likely to mount males with lower levels of sexual } \\
\text { motivation and performance (as measured by the frequency of sexual solicitations and male-to-female mounts) }\end{array}$ & Yes \\
\hline & 2b: Senescent males should be less sexually motivated and efficient than younger males & Yes \\
\hline & 2c: FMM should be more frequent in groups with a higher percentage of senescent males & Yes \\
\hline \multirow[t]{3}{*}{ 3: Few male mates } & $\begin{array}{l}\text { 3a: Female-female sexual competition for male mates should be higher in groups with more female-biased sex } \\
\text { ratios }\end{array}$ & Partly \\
\hline & 3b: FMM should mainly be performed in the context of female-female competition for male mates & Partly \\
\hline & 3c: FMM should be more frequent in groups with more female-biased sex ratios & Partly \\
\hline \multirow[t]{5}{*}{ 4: Mellow male mates } & 4a: FMM should be more frequent in groups with less frequent sexual coercion from male mates & No \\
\hline & 4b: FMM should be more frequent in groups with less intense sexual coercion from male mates & Yes \\
\hline & $\begin{array}{l}\text { 4c: FMM should be more frequent in groups where males allow their female mates to perform intermount } \\
\text { grasping behavior }\end{array}$ & Yes \\
\hline & 4d: FMM should be more frequent in groups where males are more willing to be mounted by their female mates & Yes \\
\hline & 4e: FMM should be more frequent in groups where males spent more time grooming their female mates & No \\
\hline \multirow[t]{4}{*}{ 5: Mellow third-party males } & 5a: FFM should be more frequent in groups with less frequent sexual harassment from third-party males & No \\
\hline & 5b: FFM should be more frequent in groups with less intense sexual harassment from third-party males & Yes \\
\hline & $\begin{array}{l}\text { 5c: In a group where FFM is customary, male harassment towards female homosexual consortships should be } \\
\text { less frequent than male harassment towards heterosexual consortships }\end{array}$ & No \\
\hline & $\begin{array}{l}\text { 5d: In a group where FFM is customary, male harassment towards female homosexual consortships should be } \\
\text { less intense than male harassment towards heterosexual consortships }\end{array}$ & Yes \\
\hline
\end{tabular}




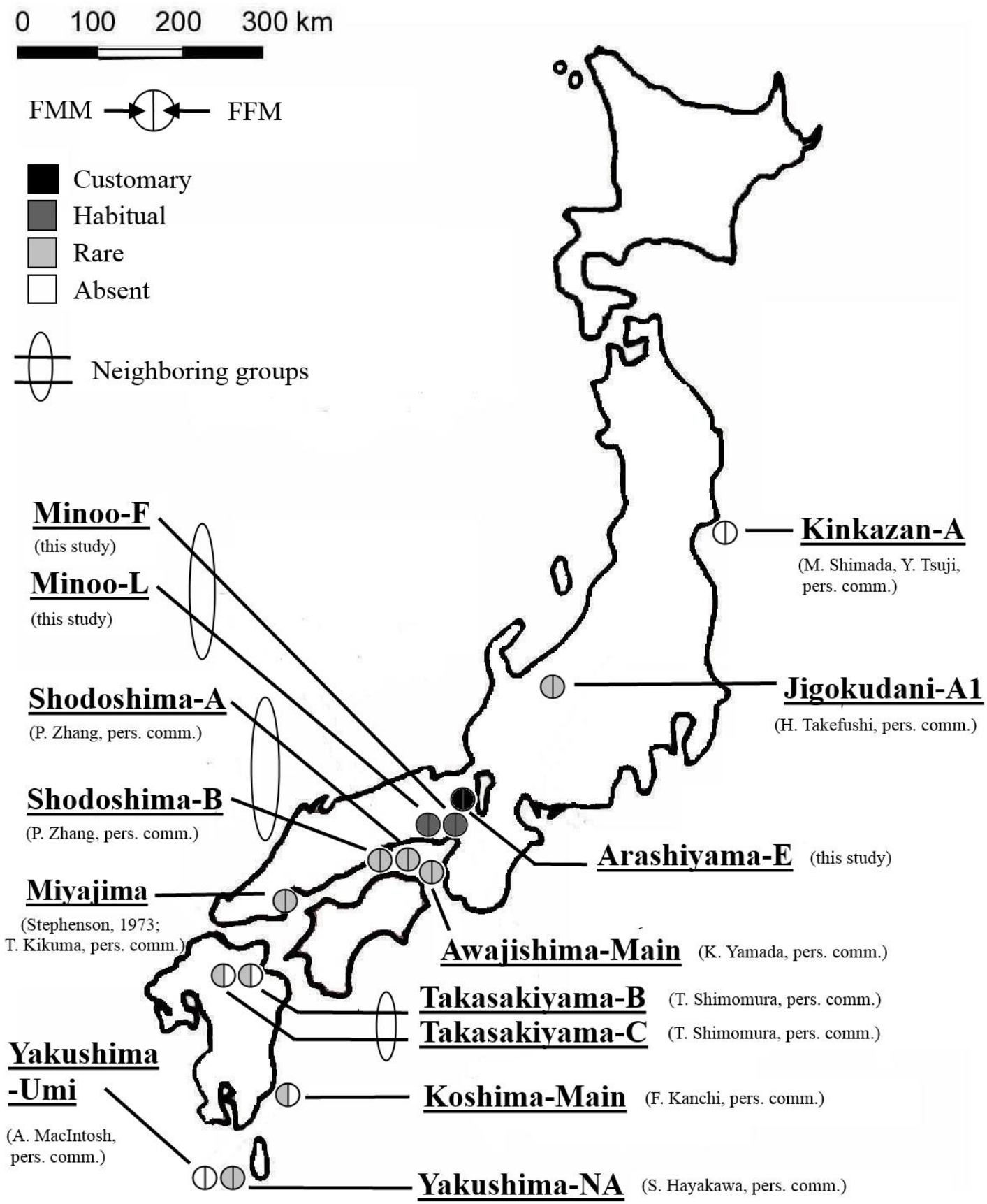

Figure 1. Map of Japan and inter-group comparison of FMM and FFM frequencies assessed by ordinal data collected during this study and complemented with a questionnaire-based survey conducted on researchers and Monkey Park staff with several years of observation during the mating season (Absent: not observed, Rare: observed from one to three times, Habitual: FMM observed from 15 to 50 times and FFM observed from 15 to 150 times, Customary: FMM observed more than 50 times and FFM observed more than 150 times; all frequency categories over at least $75 \mathrm{~h}$ of observation and weighted by the number of group members developmentally capable to engage in FMM and FFM). 
Table 2

The Four Study Groups: Composition by Age and Sex Classes of Individuals Developmentally Capable to Engage in FMM and FFM, and Data Collection Schedule Group

Arashiyama-E

$\mathrm{N}_{\text {Males+Females }}$

Males
A. Juvenile \& adolescent (2-5 yrs)
B. Young adult (6-12 yrs)
C. Middle-aged adult (13-20 yrs)
D. Senescent (> $20 \mathrm{yrs}$ )

Females

$$
\begin{aligned}
& \text { E. Adolescent (3-4 yrs) } \\
& \text { F. Young adult (5-12 yrs) } \\
& \text { G. Middle-aged adult (13-25 yrs) } \\
& \text { H. Senescent (> } 25 \mathrm{yrs})
\end{aligned}
$$

Ratios

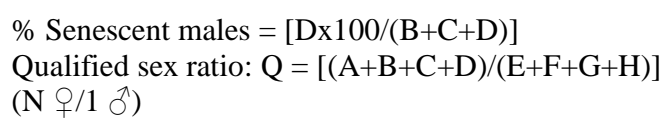

Data collection

\begin{tabular}{|c|c|}
\hline 117 & 99 \\
\hline 8 & 13 \\
\hline 1 & 7 \\
\hline 3 & 11 \\
\hline 4 & 6 \\
\hline 4 & 8 \\
\hline 25 & 23 \\
\hline 57 & 20 \\
\hline 15 & 8 \\
\hline 50.0 & 20.8 \\
\hline $\begin{array}{c}0.16 \\
6 \text { 웅ํ) }\end{array}$ & $\begin{array}{c}0.63 \\
\left(2+/ 1{ }^{\Uparrow}\right)\end{array}$ \\
\hline
\end{tabular}

Minoo-F

Minoo-L

Jigokudani-A1

66

6

12

5

19

5

3

9

3

7

10

18

16

13

14

28

9.7

$(6+/ 1$ 午)

(2ㅇ/1へ)

23.1

0.64

0.44

(2ㅇ/1
Nov.-Dec. 2012

79.4
Nov.-Dec. 2012

Oct.-Nov. 2012

Observation time (hours) 


\section{Data Collection}

The all-occurrence sampling method (Altmann, 1974) was not practicable because we studied large groups of free-ranging individuals, which rendered the continuous observation of all group members at any given time impossible. However, we used a combination of two alternative sampling methods (namely, instantaneous group scan sampling and sequence sampling) that allowed for the inter-group comparison of FMM and FFM frequencies. First, every 30 minutes, JBL conducted instantaneous group scans (Altmann, 1974) by walking from one side of the group to the other, in a set direction, and systematically recorded the occurrence of consortships. Because the means and ranges of percentages of group members sampled during scan sessions were high and similar across the four study groups (Arashiyama-E: mean \pm SD: $80.1 \pm 6.6 \%$, range: $66.9-98.3 \%, N=108$ scans; Minoo-F: mean \pm SD: $79.2 \pm 7.5 \%$, range: $66.7-99.0 \%, N=105$ scans; Minoo-L: mean \pm SD: $76.5 \pm 6.1 \%$, range: $68.2-92.4 \%$, $N=100$ scans; Jigokudani-A1: mean \pm SD: $83.7 \pm 5.0 \%$, range: $70.4-93.9 \%, N=114$ scans; chi-square goodness-of-fit test: $\chi_{3}^{2}=0.313, p=0.95$ ), we considered our inter-group comparative assessment of consortship frequency to be reliable.

Second, once a heterosexual or female homosexual consortship was recorded during a scan session, JBL, NG and LOC used the sequence sampling method (Altmann, 1974) by continuously video recording the sequence of interactions occurring during this consortship, including FMM and FFM, and all the other behavioral patterns involving the two sexual partners, as well as sexually motivated behavioral tactics employed by third-party competitors to disrupt consortships (Table 3). In Japanese macaques, FMM and FFM tend to be performed in series and occurr only within the context of conspicuous heterosexual and homosexual consortships, respectively (Vasey \& Duckworth, 2006, 2008), as opposed to single male-female or male-male mounting events that may occur outside of these interactions (e.g., dominance demonstration, tension reduction, or reconciliation). Therefore, the systematic use of this observational procedure across the four study groups allowed us to obtain reliable comparative frequencies of FMM and FFM.

A consortship was defined as a temporary, but exclusive, sexual association between two individuals, and occurred when two individuals engaged in mount-series (three or more mounts within a 10-min period) separated by intermount intervals. Consortships were deemed to have terminated if the two partners were not in proximity (separated by a distance of more than $1 \mathrm{~m}$ ) and exhibited no mounting for $10 \mathrm{~min}$ ( $c f$. Vasey, 2004). Intense sexual coercion and sexual harassment included agonistic behaviors with body contact (i.e., pushing, hitting, grabbing, and biting). The visibility conditions were generally excellent at the three sites. The data collection schedule and observation time for each group are provided in Table 2.

These continuous data from four groups were complemented with ordinal data from 10 additional groups collected via a questionnaire-based survey conducted on local researchers and Monkey Park staff with at least three years of observation during the mating season (Figure 1). These ordinal data consisted of four frequency categories of FMM and FFM (observed over a 75h period, and weighted by the number of group members developmentally capable to engage in such mounts), namely absent: not observed, rare: observed from one to three times, habitual: FMM observed from 15 to 50 times and FFM observed from 15 to 150 times, and customary: FMM observed more than 50 times and FFM observed more than 150 times. 
Leca et al. 394

Table 3

Social and Sexual Behavioral Patterns Collected During the Study

\begin{tabular}{ll}
\hline Behavioral patterns & Description \\
\hline Sexual solicitation & Body postures (frozen stance and intense gazing, hindquarter presentations, inclined-back presentations, and crouching while \\
screaming), body movements and gestures (lip quivering, head bobbing, ground smacking, hindquarter sniffing, hands-on- \\
hindquarters solicitations, pushing, grabbing, body spasms, and repeated glancing while sitting with 10 m of the target), and sexual \\
vocalizations
\end{tabular}

Double foot-clasp mount

Reclining mount

Sitting mount

Standing mount

Ventro-ventral mount

Pelvic thrusting

Intermount grasping behavior

Intermount allogrooming behavior Sexual coercion

Sexual harassment

Female-female competition for male mates
Mounter grasps with his or her feet between the mountee's ankles and hips, and with his or her hands on the mountee's back

Mounter lays ventrally on the mountee's back, using his or her feet to grasp the mountee's legs above the ankles and his or her hands to grasp fur on the mountee's upper back

Mounter sits on the mountee's back in a jockey-like position, while grasping the mountee's upper back with his or her hands and the mountee's lower back with his or her feet (Figure 4)

Mounter grasps from behind the mountee's back with its hands, while either standing bipedally with his or her feet on the ground and his or her knees slightly bent, or standing with one foot on the ground and the other grasping the mountee's hind limb

Both participants embrace each other with their arms and legs, with chest-to-chest and belly-to-belly contact, facing each other in a standing, sitting, or lying position

Rhythmic movements of the mounter's pelvic girdle toward the mountee's perineum

One consort partner holds the other while sitting in dorso-ventral orientation (i.e., both partners in a sitting position and belly-to-back contact)

One consort partner removes ectoparasites from the hair and body surface of the other

Male consort partner alternatively sexually solicits and aggresses his female mate

Third-party male interrupts a consortship by displacing or aggressing both consortship partners

Third-party female approaches a heterosexual consortship and solicits the male consort partner 


\section{Data Analysis}

JBL scored all of the video records. When applicable, we weighted behavioral frequencies by considering demographic variation between groups, i.e., group-specific numbers of individuals being developmentally capable to engage in FMM and FFM (Table 2; $c f$. Leca, Gunst, \& Vasey, in press). To assess inter-group differences in the cumulative frequency of FMM over the same observation time, we multiplied the cumulative number of FMM observed in group $i$ by $\overline{\mathrm{x}}_{\mathrm{FM}} / \mathrm{x}_{\mathrm{FM}} i$, with $\overline{\mathrm{x}}_{\mathrm{FM}}=$ mean number of females and males being developmentally capable to engage in FMM across the four study groups and $\mathrm{x}$ FM $i=$ number of females and males being developmentally capable to engage in FMM in group $i$. To assess inter-group differences in the cumulative frequency of FFM over the same observation time, we multiplied the cumulative number of FFM observed in group $i$ by $\overline{\mathrm{x}}_{\mathrm{F}} / \mathrm{x}_{\mathrm{F}} i$, with $\overline{\mathrm{x}}_{\mathrm{F}}=$ mean number of females being developmentally capable to engage in FFM across the four study groups and $\mathrm{x}_{\mathrm{F} i}=$ number of females being developmentally capable to engage in FFM in group $i$. By doing so, we gave more weight to groups with fewer members being developmentally capable to engage in FMM and FFM, and vice versa. We used the same weighting method to assess inter-group differences in the frequency of female-to-male and female-to-female solicitations and the frequency of female-female competition for male mates.

Other behavioral frequencies (e.g., intermount grasping behavior, intermount allogrooming behavior, and sexual coercion; Table 3) were defined as the number of behavioral events per hour of consortship. FMM and FFM prevalence was defined as the percentage of female group members performing FMM and FFM, respectively. Because our raw and transformed data violated parametric assumptions, we conducted non-parametric tests. When investigating inter-group differences in the cumulative frequencies of FMM and FFM, with no specification of the predicted directions of the differences (Table 4), we used two-tailed tests. Because all of the other analyses were motivated by clear directional predictions - they indicated the predicted directions of the inter-group differences; $c f$. Table 1 - we used one-tailed tests (Siegel \& Castellan, 1988). Statistical analyses were performed using the SPSS 13.0 analytical program. Significance levels were set at $\alpha=0.05$.

Table 4

Inter-group Differences in the Weighted Cumulative Frequencies of FMM and FFM (two-tailed Kolmogorov-Smirnov tests)

\begin{tabular}{lcc} 
Inter-group differences & \multicolumn{2}{c}{ Type of female mounting behavior } \\
\hline & FMM & FFM \\
Arashiyama-E $>$ Minoo-F & $\mathrm{z}=2.008, p=0.001$ & $\mathrm{z}=2.008, p=0.001$ \\
Arashiyama-E > Minoo-L & $\mathrm{z}=1.643, p=0.009$ & $\mathrm{z}=2.008, p=0.001$ \\
Arashiyama-E > Jigokudani-A1 & $\mathrm{z}=2.739, p<0.001$ & $\mathrm{z}=2.739, p<0.001$ \\
Minoo-F = Minoo-L & $\mathrm{z}=1.095, p=0.181$ & $\mathrm{z}=0.548, p=0.925$ \\
Minoo-F $>$ Jigokudani-A1 & $\mathrm{z}=2.191, p<0.001$ & $\mathrm{z}=2.191, p<0.001$ \\
Minoo-L > Jigokudani-A1 & $\mathrm{z}=2.373, p<0.001$ & $\mathrm{z}=2.191, p<0.001$ \\
\hline
\end{tabular}

\section{Results}

\section{“Female Mounting Covariation" Hypothesis}

FMM and FFM frequency. We found marked inter-group differences in the total number of FMM and FFM over 75 hours of observation (Arashiyama-E: 80 FMM and 277 FFM; Minoo-F: 20 FMM and 39 FFM; Minoo-L: 19 FMM and 28 FFM; Jigokudani-A1: 0 FMM and 0 FFM). After weighting these data by considering demographic variation between groups, we found statistically significant intergroup differences in the cumulative frequencies of FMM and FFM over 75 hours of observation (Figure 
2; Table 4). Such differences allowed us to distinguish between three categories of study groups: (1) a group like Arashiyama-E, in which FMM and FFM were customary, (2) groups like Minoo-F and MinooL, in which FMM and FFM were habitual, and (3) a group like Jigokudani-A1, in which FMM and FFM did not occur during the study period.

When considering continuous data, we found a significant positive correlation between FMM and FFM frequencies across our four study groups (Spearman rank-order correlation: $\mathrm{R}_{\mathrm{s}}=1.00, N=4, p<$ 0.001). When considering ordinal data, we also found a significant positive correlation between FMM and FFM frequencies across 14 free-ranging groups of Japanese macaques (Kendall rank-order correlation: $\mathrm{T}_{\mathrm{b}}$ $=0.80, N=14, p=0.001$; Figure 1). Out of 14 groups, 11 showed identical frequency categories of FMM and FFM (ranging from absent to customary), and three showed minor differences in frequency categories (i.e., FMM was rare and FFM was absent). The three pairs of neighboring groups showed identical frequency categories of FMM and FFM. Therefore, Prediction \# 1a was supported. In three groups, FMM was observed, but FFM was not. In none of the groups surveyed was FFM observed in the absence of FMM.

A)

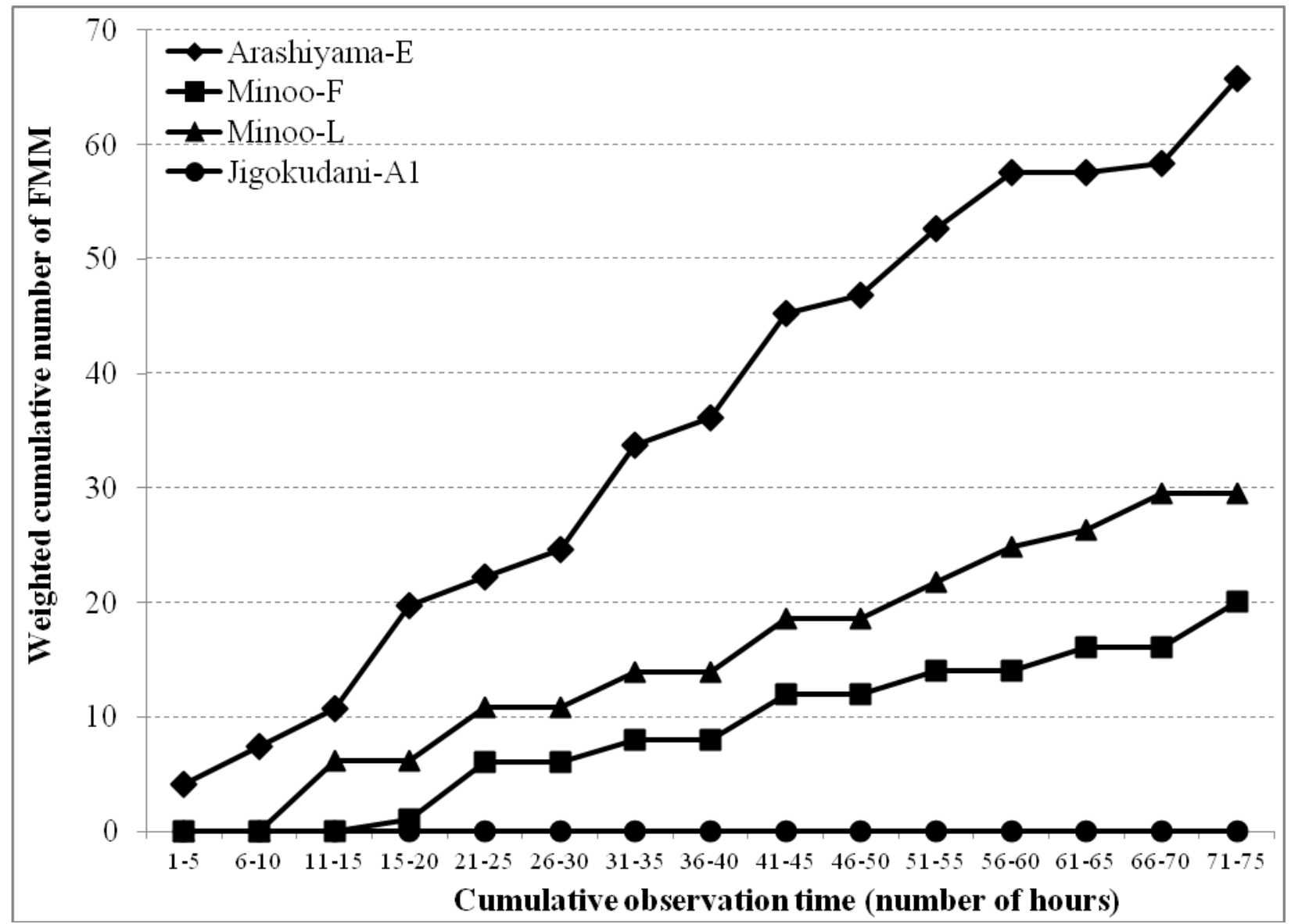


B)

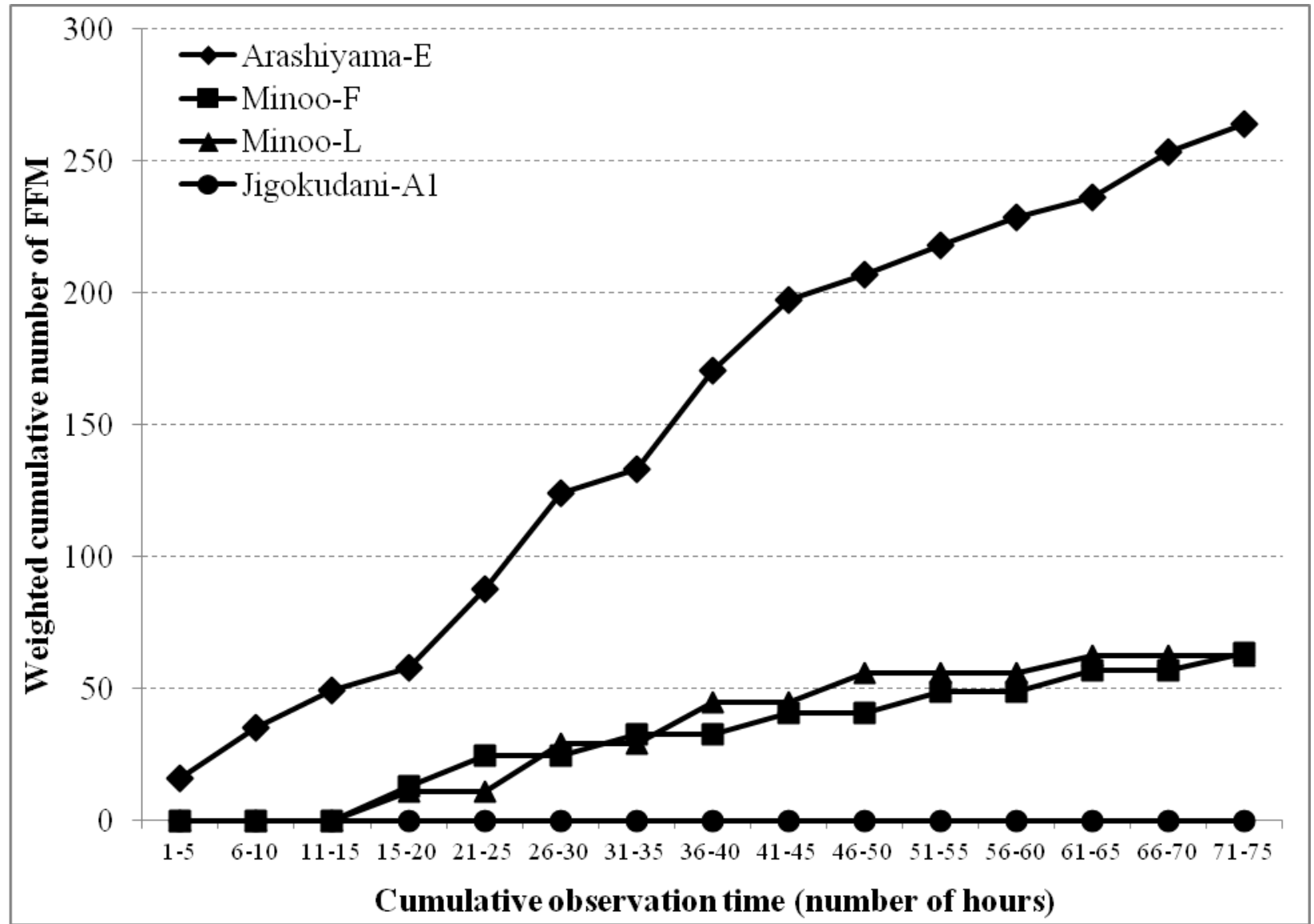

Figure 2. A) Inter-group differences in the cumulative number of FMM observed (weighted by group-specific numbers of females and males being developmentally able to engage in FMM) over the study period. B) Inter-group differences in the cumulative number of FFM observed (weighted by group-specific numbers of females being developmentally able to engage in FFM) over the study period.

FMM and FFM prevalence. Arashiyama-E showed the highest prevalence of both FMM and FFM (9.8 and 28.4\%, respectively) and Jigokudani-A1 showed the lowest prevalence of both FMM and FFM (0 and $0 \%$, respectively). As predicted, Minoo-F and Minoo-L had intermediate prevalence values for both FMM and FFM. However, because Minoo-F and Minoo-L showed minor discrepancies from the expected pattern (FMM prevalence: 8.1 and 8.5\%, respectively; FFM prevalence: 11.3 and 10.6\%, respectively), the positive correlation between FMM and FFM prevalence did not reach statistical significance $\left(\mathrm{R}_{\mathrm{s}}=0.80, N=4, p=0.100\right)$. The fact that the test reached statistical significance when computed with numbers (instead of percentages) of females $\left(\mathrm{R}_{\mathrm{s}}=1.00, N=4, p<0.001\right)$ suggests a small sample size effect. Therefore, Prediction \#1b was partly supported.

In the Arashiyama-E group, only two females performed both FMM and FFM. This represents $20 \%$ of females performing FMM and $7 \%$ of females performing FFM. In the Minoo-F group, only one female performed both FMM and FFM. This represents 20\% of females performing FMM and 14\% of females performing FFM. In the Minoo-L group, only one female performed both FMM and FFM. This represents $25 \%$ of females performing FMM and $20 \%$ of females performing FFM. Even within age classes, the percentage of females performing FMM and the percentage of females performing FFM differed significantly (Table 5). Therefore, Prediction \#1c was not supported. 
Table 5 .

Percentage of FMM and FMM Performed by Females of Different Age Classes Across Study Groups

\begin{tabular}{|c|c|c|c|c|c|c|}
\hline \multirow[b]{2}{*}{ Group } & \multirow[b]{2}{*}{ Type of mount } & \multicolumn{4}{|c|}{ Female age class } & \multirow[b]{2}{*}{$\begin{array}{c}\text { Fischer's exact } \\
\text { test }(p)\end{array}$} \\
\hline & & Adolescent & Young adult & $\begin{array}{c}\text { Middle-aged } \\
\text { adult }\end{array}$ & Senescent & \\
\hline \multirow{2}{*}{ Arashiyama-E } & FMM & 0 & 9 & 86 & 5 & \multirow{2}{*}{$<0.001$} \\
\hline & FFM & 18 & 53 & 18 & 11 & \\
\hline \multirow{2}{*}{ Minoo-F } & FMM & 0 & 5 & 30 & 65 & \multirow{2}{*}{$<0.001$} \\
\hline & FFM & 74 & 26 & 0 & 0 & \\
\hline \multirow{2}{*}{ Minoo-L } & FMM & 0 & 0 & 32 & 68 & \multirow{2}{*}{$<0.001$} \\
\hline & FFM & 82 & 18 & 0 & 0 & \\
\hline
\end{tabular}

Form of FMM and FFM. At Arashiyama, where FMM and FFM were customary: (1) All five major female mounting postures were observed with similar percentages for FMM and FFM (Figure $3 ; \chi_{4}^{2}$ $=5.81, p=0.214)$, (2) pelvic thrusting was relatively frequent and occurred at similar percentages for FMM and FFM (31.3 and 42.2\%, respectively; $\left.\chi^{2}{ }_{1}=3.13, p=0.077\right)$, (3) both male and female mountees actively solicited female mounting (e.g., via hindquarter and/or inclined-back presentations or arm movement directed to the female), (4) in both heterosexual and female homosexual consortships, bidirectional mounts were relatively frequent ( $61 \%$ of heterosexual consortships and $69 \%$ of homosexual consortships), and occurred during the entire duration of consortships, and (5) male mountees during FMM belonged to all age classes, with substantial percentages in all age class (young adult: 16\%, middleaged adult: $18 \%$, and senescent: $66 \%$ ) and female mountees during FFM belonged to all age classes (adolescent: 18\%, young adult: 52\%, middle-aged adult: 18\%, and senescent: 12\%).

At Minoo, where FMM and FFM were habitual (i.e., less frequent than at Arashiyama): (1) fewer types of female mounting postures were observed with similar percentages for FMM and FFM (Figure 3; Minoo-F: four mounting postures, $\chi_{3}^{2}=4.138, p=0.250$; Minoo-L: three mounting postures, $\chi_{2}^{2}=1.68, p$ $=0.431$ ), (2) pelvic thrusting was relatively rare and occurred at similar percentages for FMM and FFM (Minoo-F: 15.0 and 25.6\%, respectively; $\chi_{1}^{2}=0.87, p=0.351$; Minoo-L: 10.5 and $17.8 \%$, respectively; $\chi_{1}^{2}=0.48, p=0.488$ ), (3) neither male nor female mountees actively solicited female mounting, (4) in both heterosexual and female homosexual consortships, bidirectional mounts were very occasional (Minoo-F: 12\% and 23\%, respectively; Minoo-L: 7\% and 11\%, respectively), (5) FMM only occurred at the beginning of heterosexual consortships (i.e., before males started their mount series), and (6) although male mountees during FMM belonged to all age classes, the senescent age class was overrepresented (Minoo-F: young adult: 10\%, middle-aged adult: 10\%, and senescent: $80 \%$; Minoo-L: young adult: 10\%, middle-aged adult: 5\%, and senescent: $85 \%$ ) and female mountees during FFM belonged to only two age classes (Minoo-F: adolescent: 74\%, young adult: 26\%; Minoo-L: adolescent: 82\%, young adult: 18\%).

Taken together, these results suggest that structural diversity of FMM and FFM (i.e., female mounting postures, directionality, and timing, as well as pelvic movement) was positively correlated across groups. Therefore, Prediction \#1d was supported. 


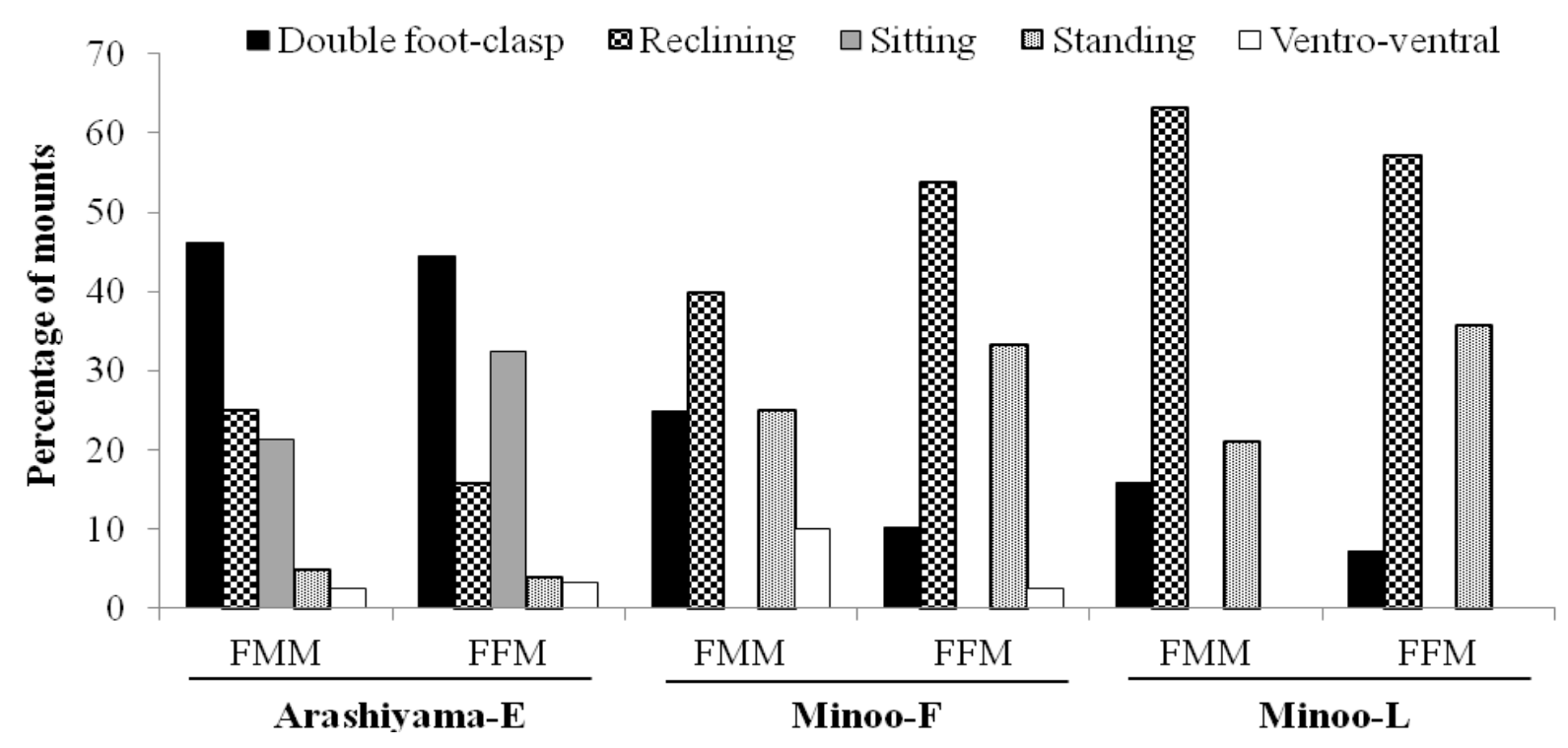

Figure 3. Inter-group differences in the percentage of mounting postures (FMM and FFM).

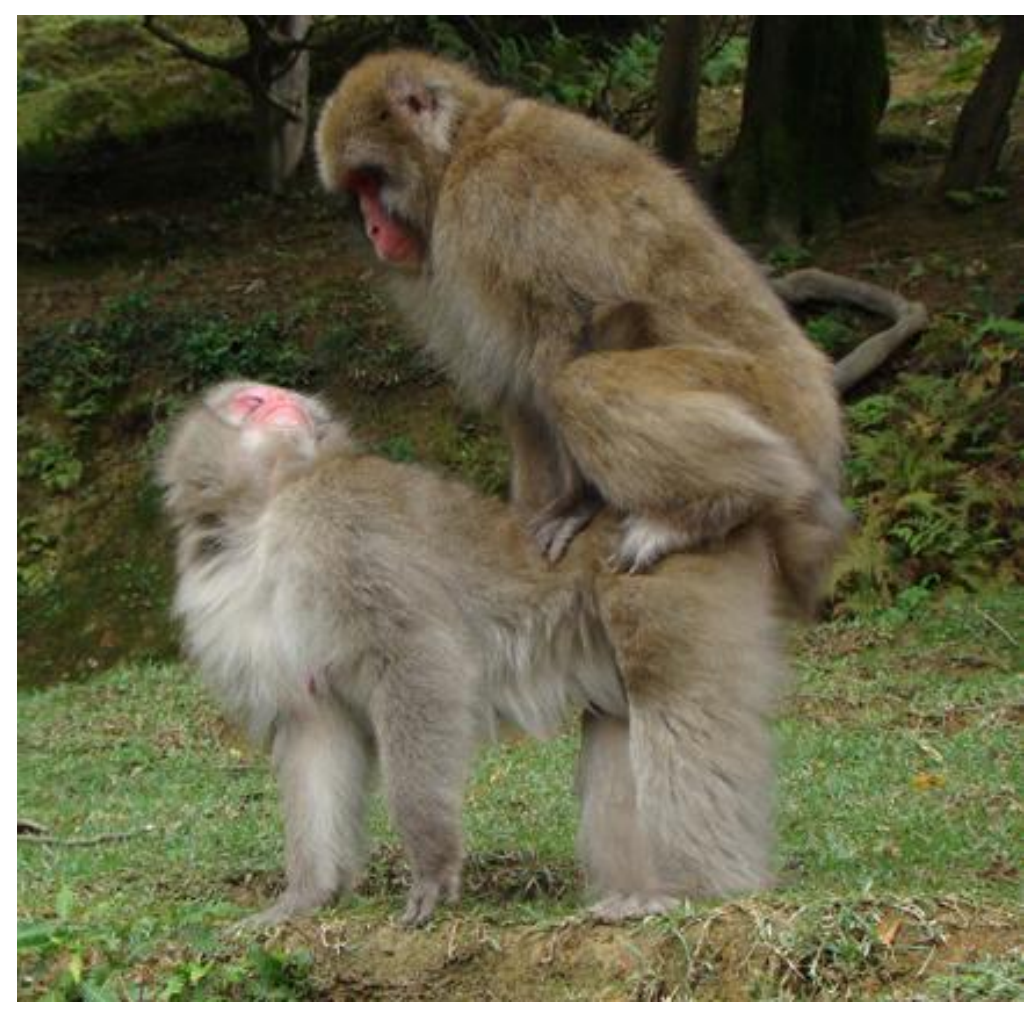

Figure 4. Sitting mount posture during FFM at Arashiyama (photo by J.-B. Leca).

\section{“Senescent Male Mates" Hypothesis}

In the three groups where FMM occurred, females were significantly more likely to mount males (1) that solicited them less frequently (Mann-Whitney tests, Arashiyama-E: $z=-2.40, p=0.017$; Minoo$\mathrm{F}: z=0-2.98, p=0.003$; Minoo-L: $z=-3.01, p=0.003$ ), and (2) that mounted them less frequently 
(Arashiyama-E: $z=-2.02, p=0.043$; Minoo-F: $z=-1.98, p=0.048$; Minoo-L: $z=-2.91, p=0.004$ ). Therefore, Prediction \#2a was supported.

In the four study groups, we found significant male age differences in the frequency of male-tofemale sexual solicitations (Kruskal-Wallis tests, Arashiyama-E: $\chi_{2}^{2}=18.46, p<0.001$; Minoo-F: $\chi_{2}^{2}=$ 21.36, $p<0.001$; Minoo-L: $\chi_{2}^{2}=31.08, p<0.001$; Jigokudani-A1: $\chi_{2}^{2}=17.21, p<0.001$ ) and male-tofemale mounting (Arashiyama-E: $\chi_{2}^{2}=34.55, p<0.001$; Minoo-F: $\chi_{2}^{2}=38.97, p<0.001$; Minoo-L: $\chi_{2}^{2}=$ $36.59, p<0.001$; Jigokudani-A1: $\left.\chi_{2}^{2}=34.65, p<0.001\right)$. In each group, senescent males solicited and mounted females at significantly lower frequencies than young adult males $(p<0.05)$ and middle-aged adult males $(p<0.05)$, whereas the latter two did not significantly differ. Therefore, Prediction \#2b was supported.

We found a significant positive correlation between FMM frequency and the percentage of senescent males across groups $\left(\mathrm{R}_{\mathrm{s}}=1.00, N=4, p<0.001\right)$. Therefore, Prediction \#2c was supported.

Finally, we found a significant inter-group difference in the frequency of female-to-male sexual solicitations, not including FMM (Kruskal-Wallis test, $\chi_{2}^{2}=20.70, p<0.001$ ). Multiple paired comparisons based on mean ranks showed that female-to-male sexual solicitations were significantly more frequent in Arashiyama-E (i.e., the group with the highest percentage of senescent males) than in the Minoo-F, Minoo-L, and Jigokudani-A1 groups $(p<0.05)$, whereas the latter three did not significantly differ.

\section{"Few Male Mates" Hypothesis}

The group with the most heavily female-biased sex ratio (i.e., Arashiyama-E: $\mathrm{Q}=0.16, c f$. Table 2) showed the highest frequency of female-female competition for male mates (mean $=4.3 \pm 7.1$ competition events/hour). The group with the least heavily female-biased sex ratio (i.e., Jigokudani-A1: Q $=0.64$ ) showed the lowest frequency of female-female competition for male mates (mean $=0.4 \pm 1.2$ competition events/hour). As predicted, Minoo-F and Minoo-L had intermediate values for both variables. However, because Minoo-F $(\mathrm{Q}=0.63$; mean $=1.3 \pm 2.2$ competition events/hour $)$ and Minoo-L $(\mathrm{Q}=0.44$; mean $=1.2 \pm 1.5$ competition events/hour) showed minor discrepancies from the expected pattern, the negative correlation between $\mathrm{Q}$ and female-female competition did not reach statistical significance $\left(\mathrm{R}_{\mathrm{s}}=\right.$ $-0.80, N=4, p=0.100)$. Therefore, Prediction \#3a was partly supported.

In the Arashiyama-E group, FMM was more likely to occur during consortships with higher female-female competition for male mates (Mann-Whitney test, $z=-4.59, p<0.001$ ). Although we found similar tendencies in Minoo-F and Minoo-L, they did not reach statistical significance (Minoo-F: $z=-$ $1.42, p=0.155$; Minoo-L: $z=-1.74, p=0.081$ ). Therefore, Prediction \#3b was partly supported.

The group with the most heavily female-biased sex ratio (i.e., Arashiyama-E: $Q=0.16$ ) showed the highest FMM frequency (1.02 FMM/hour). The group with the least heavily female-biased sex ratio (i.e., Jigokudani-A1: $\mathrm{Q}=0.64)$ showed the lowest FMM frequency $(0.00 \mathrm{FMM} /$ hour $)$. However, due to minor discrepancies between Minoo-F $(\mathrm{Q}=0.63 ; 0.25$ FMM/hour $)$ and Minoo-L $(\mathrm{Q}=0.44 ; 0.24$ FMM/hour), the negative correlation between $\mathrm{Q}$ and FMM frequency did not reach statistical significance $\left(\mathrm{R}_{\mathrm{s}}=-0.80, N=4, p=0.100\right)$. Therefore, Prediction \#3c was partly supported.

\section{"Mellow Male Mates" Hypothesis}

We found no significant correlation between the frequency of FMM and the frequency of all sexual coercive acts performed by male mates across groups $\left(\mathrm{R}_{\mathrm{s}}=-0.80, N=4, p=0.100\right)$. The Arashiyama-E group had the highest FMM frequency but not the lowest frequency of sexual coercion. Therefore, Prediction \#4a was not supported. In contrast, we found a significant negative correlation between the frequency of FMM and the frequency of intense sexual coercive acts performed by male mates $\left(\mathrm{R}_{\mathrm{s}}=-1.00, N=4, p<0.001\right)$. Therefore, Prediction \#4b was supported.

We found a significant positive correlation between FMM frequency and the time spent performing female-to-male intermount grasping behavior across groups $\left(\mathrm{R}_{\mathrm{s}}=1.00, N=4, p<0.001\right)$. At 
Arashiyama, where FMM was customary, females performed intermount grasping behavior towards all males of all age classes. At Minoo, where FMM was habitual, females performed this behavior only towards senescent males (Minoo-F: towards 4 out 5 senescent males engaged in consortships; Minoo-L: towards 2 out of 3 senescent males engaged in consortships). At Jigokudani, where FMM was not recorded during the study period, we did not observe female-to-male intermount grasping behavior. Therefore, Prediction \#4c was supported.

That being said, we found no significant intergroup differences in how often male mates rebuffed females' attempts to perform intermount grasping behavior (Kruskal-Wallis test, $\chi_{3}^{2}=4.80, p=0.187$ ). As such, although FMM was more frequent in groups where female-to-male intermount grasping behavior was more often performed, lack of female-to-male intermount grasping was not associated with males' aggression toward females that attempted to perform this behavior.

There was a significant intergroup difference in how often males actively solicited FMM (e.g., via back presentations or arm movement directed to the female; Kruskal-Wallis test, $\chi_{3}^{2}=31.19, p<$ 0.001). Such behavioral patterns were significantly more frequent at Arashiyama (where FMM was customary) than in the Minoo-F, Minoo-L, and Jigokudani-A1 groups $(p<0.05)$, whereas the latter three did not significantly differ (noteworthy, male solicitations of FMM were absent in these groups). Therefore, Prediction \#4d was supported.

We found a significant intergroup difference in the time spent performing male-to-female grooming behavior during intermount intervals (Kruskal-Wallis test, $\chi_{3}^{2}=23.91, p<0.001$ ). However, the direction of significant paired comparisons was contrary to our predictions. Males spent more time grooming their female mates at Minoo and Jigokudani than at Arashiyama, and more time at Minoo than at Jigokudani $(p<0.05)$. There was no significant difference between Minoo-F and Minoo-L in terms of male-to-female grooming behavior during intermount intervals. Therefore, Prediction \#4e was not supported.

\section{“Mellow Third-Party Males" Hypothesis}

There were no significant negative correlations (1) between the frequency of all male sexual harassment towards heterosexual consortships and the frequency of FFM across groups $\left(\mathrm{R}_{\mathrm{s}}=-0.20, N=4\right.$, $p=0.400$ ), and (2) between the frequency of all male sexual harassment towards homosexual consortships and the frequency of FFM and across groups $\left(\mathrm{R}_{\mathrm{s}}=0.50, N=3, p=0.333\right)$. Therefore, Prediction \#5a was not supported.

However, we found significant negative correlations (1) between the frequency of intense male sexual harassment towards heterosexual consortships and the frequency of FFM across groups $\left(\mathrm{R}_{\mathrm{s}}=-1.00\right.$, $N=4, p<0.001$ ), and (2) between the frequency of intense male sexual harassment towards homosexual consortships and the frequency of FFM and across groups $\left(\mathrm{R}_{\mathrm{s}}=-1.00, N=3, p<0.001\right)$. Therefore, Prediction \#5b was supported.

At Arashiyama, where FFM was customary, the frequencies of all male sexual harassment towards homosexual consortships and towards heterosexual consortships did not differ significantly (Mann-Whitney test, $z=-0.76, p=0.447$ ). Therefore, Prediction \#5c was not supported. However, the frequency of intense male sexual harassment towards homosexual consortships was significantly lower than that towards heterosexual consortships $(z=-2.15, p=0.031)$. Therefore, Prediction \#5d was not supported.

\section{Discussion}

\section{Explaining Inter-Group Differences in Female Mounting}

Our study revealed marked inter-group differences in the frequency, prevalence, and form of FMM and FFM in Japanese macaques (Figure 1). We also found that these two types of non-conceptive mounting covaried in their frequency and form across groups (Table 1). In other words, groups exhibiting 
high levels of, and structurally diverse, FMM also showed high levels of, and structurally diverse, FFM, and vice versa. Because the duration of observations at Jigokudani was about twice that at Arashiyama and Minoo (Table 2), failure to record FMM and FFM in the Jigokudani-A1 group during our study period could not be attributed to a lack of research effort. Indeed, thirty years of routine observation at the Jigokudani Monkey Park showed that (1) both FMM and FFM were extremely rare in this group, (2) the very few cases of FMM were performed by middle-aged and old females, (3) the very few cases of FFM were performed by adolescent and young adult females, and (4) the structural diversity of FMM and FFM was very low, with only one mounting posture being observed: reclining mounts (H. Takefushi, pers. comm.; Figure 1). Although this information is anecdotal, it is consistent with the "female mounting covariation" hypothesis.

From a developmental perspective, at Arashiyama and Minoo, the majority of FFM were performed by adolescent and young adult females, whereas the majority of FMM were performed by middle-aged and senescent females (Table 5). Two social factors can explain why FFM appears earlier and develops more rapidly than FMM in the ontogeny of Japanese macaques. First, the presence of motivated same-sex sexual partners may furnish a social context in which young females could practice FFM, whereas in the absence of motivated opposite-sex partners no such context exists (Leca et al., in review). Second, adolescent females received more intense aggressions during heterosexual interactions than during homosexual ones and severe aggressions resulting in wounds are more likely to be lifethreatening on smaller-bodied adolescent females than on larger-bodied adult females. Consequently, young females may prefer to engage in safer homosexual interactions (including FFM) in lieu of more risky heterosexual ones, including FMM (Gunst, Leca, \& Vasey, in review). The latter argument could explain why Prediction \#5d was supported and Prediction \#1c was not.

From an evolutionary perspective, Vasey (2007) argued that if adult FFM in Japanese macaques was a by-product of adult FMM, then the latter should be more phylogenetically primitive than the former. As such, there might be groups with FMM and no FFM, but there should not be groups with FFM and no FMM. Our results are congruent with this view (Figure 1). In three groups (i.e., Takasakiyama-B, Takasakiyama-C and Koshima-Main) FMM has been observed but FFM has not. At none of the sites surveyed did FFM exist in the absence of FMM. In addition, Vasey (2007) predicted that FFM would occur only in groups where females obtained immediate sexual reward via genital stimulation. Although we did not undertake a detailed test of this hypothesis, the fact that pelvic thrusting occurred during FFM at all three sites where FFM was observed is consistent with this view. Taken together, our findings support the "female mounting covariation" hypothesis, and provide further evidence that FMM and FFM in Japanese macaques are developmentally and evolutionarily linked (see also Leca et al., in review; Vasey, 2007).

In our attempt to account for inter-group differences in the frequency of FMM, we provided some support to each of the three socio-demographic hypotheses tested (Table 1). First, we found that frequent FMM at the group level was associated with a high percentage of senescent (hence sexually undermotivated) male group members (for example, at Arashiyama, Table 2). That senescent males were less sexually motivated and efficient than younger males (as measured by the frequency of sexual solicitations and male-to-female mounts) is consistent with previous research on the ontogeny of sexual performance in male primates (Alberts, 2012). This result could partly be explained by age-related dominance. Because old male Japanese macaques generally rank higher than young ones (Sprague, 1998), their consortships are less likely to be targeted by third-party males. Facing lower male intra-sexual competition, old and dominant males may be less pressured than young and subordinate males to solicit and mount their female mates frequently. In turn, their female consort partners may be more pressured to stimulate them by performing more frequent FMM, particularly in the context of high female-female competition for male mates, as was the case at Arashiyama.

Lower sexual motivation on the part of senescent males could also be explained, in part, by factors related to physiological aging. For example, senescent male Japanese macaques exhibit weaker penile erection and lower rates of penile intromission than younger males during heterosexual consortships ( $c f$. Alberts, 2012). As a result, their female mates may obtain lower levels of sexual rewards 
and may be more likely to perform FMM in order to stimulate their genital area (Vasey \& Duckworth, 2008), as they would do with female sexual partners during homosexual consortships (Vasey \& Duckworth, 2006). Overall, our results strongly supported the "senescent male mates" hypothesis.

Second, the "few male mates" hypothesis received partial support (Table 1). In the Arashiyama-E group, the heavily female-biased sex ratio led to a high level of female-female competition for male mates, which in turn, was associated with frequent FMM. This is consistent with the view that FMM could serve to focus the male's attention and prevent him from moving toward female competitors who are soliciting him for sex. In the Jigokudani-A1 group, we found a more balanced sex ratio, hence lower female-female competition for male mates, which may explain the absence/scarcity of FMM. At Minoo, the situation was intermediate, although Minoo-F and Minoo-L did not exactly comply with this pattern.

Third, we found that FMM was more tolerated in groups with less coercive and less controlling male mates, as measured by fewer intense sexual coercive acts, more female-to-male intermount grasping behavior, and more male-to-female solicitations to expedite FMM. Female primates engaged in heterosexual consortships are easy targets of repeated and sometimes severe aggressions inflicted by their male mates (Muller \& Wrangham, 2009). This may explain why FMM - a sexual interaction that involves close contact and coordination with established male mates who have demonstrated a degree of trustworthiness - is relatively rare in Japanese macaques, a species characterized by intense and asymmetrical aggressive interactions (Thierry, Iwaniuk, \& Pellis, 2000). At Jigokudani, and to a lesser extent at Minoo, the intensity of male-to-female sexual coercive acts may prevent females from performing FMM. When male-female conflicts are less intense, like at Arashiyama, FMM may be expressed more frequently. We argue that such a rationale can be generalized to explain some interspecies differences in sexual behavior. For example, the virtual absence of male sexual coercion of female bonobos (Paoli, 2009) may account for the species' hypersexuality, including various forms of sexual interactions involving individuals of all age and sex classes, and close association between males and females throughout the female reproductive cycle. Overall, our results support the "mellow male mates" hypothesis.

FFM is a showy signal of sexual interest that attracts the attention of multiple third-party individuals. As such, partners engaged in a FFM are at increased risk of attack from third-party competitors. Given this, it is not surprising that FFM was more tolerated in groups with lower levels of sex-related aggressiveness by third-party males, as measured by fewer cases of intense male sexual harassment. At Arashiyama, male sexual harassment towards female homosexual consortships was less intense than that towards heterosexual consortships. This may account, in part, for the frequent occurrence and long-term maintenance of FFM in this group. In contrast, high levels of intense sexual harassment towards females engaged in heterosexual and homosexual consortships may be responsible, in part, for the infrequent occurrence of FFM at Minoo and its (virtual) absence at Jigokudani.

Over approximately $150 \mathrm{hrs}$ of observation at Minoo and at Jigokudani during the mating season, we recorded numerous cases of physical injuries incurred by adolescent and adult females engaged in sexual interactions, including open wounds resulting in bleeding and internal wounds resulting in limping for at least one day (Minoo-F: 7 cases, $N=57$ females, Minoo-L: 7 cases, including one leading to the female's death, $\mathrm{N}=41$ females, Jigokudani-A1: 12 cases, $\mathrm{N}=66$ females). In comparison, we recorded only 9 cases of physical injuries and none of which were deadly in the Arashiyama-E group in a total of 508 hrs of observation over two consecutive mating seasons $(N=101$ females; Leca \& Gunst, unpublished data). Moreover, we noticed that most female homosexual consortships at Arashiyama occurred either within the core of the group or in plain view in front of other groups members, whereas most female homosexual consortships occurred either at the periphery of the group or were half-hidden behind the vegetation at Minoo (Leca, personal observation). These results complement a previous study at Arashiyama showing that adult males' disinterest in adolescent females' sexual solicitations and male aggressive behavior towards young females are key to explain the development of FFM in Japanese macaques (Gunst et al., in review). The present study suggests that the intensity of male sexual harassment towards all resident females may account, in part, for inter-group differences in FFM. Taken together, our data support the "mellow third-party males" hypothesis. 
The latter four hypotheses are not mutually exclusive. The customary occurrence, high prevalence, and great diversity of FMM and FFM in the Arashiyama-E group are likely to be the result of combined favorable socio-demographic conditions, namely few resident males, most of them being old, sexually under-motivated, and less aggressive and controlling than younger male Japanese macaques in their sexual prime. When all these conditions are met, we argue that FMM and FFM may be expressed and maintained within a group. It is noteworthy that some of these conditions may covary. First, senescent males are not only sexually under-motivated (which could trigger FMM), but they are also slower when it comes to chasing and catching up with females (which could result in less intense sexual coercion and harassment; $c f$. Goodall, 1986, p. 481). Second, due to the heavily female-biased sex ratio in the Arashiyama-E group, males may not have to be overtly aggressive to gain access to female mates because fewer competitors are present and females may be more proceptive. When all of these conditions are not met, like at Minoo and to a greater extent at Jigokudani, the expression of FMM and FFM may be tempered or suppressed altogether, or their long-term maintenance within the group may be jeopardized. As such, demographic and sociocultural hypotheses are compatible.

Overall, our results are in agreement with the view that group composition is a major factor contributing to our understanding of the expression of sexual behavior and mating strategies ( $c f$. Goldizen, 1988). Even though our findings reveal clear group-specific profiles in the occurrence and expression of non-conceptive sexual activity in female Japanese macaques, we believe they are not incompatible with more flexible "situational" homosexuality expressed by human males and females under a variety of same-sex settings, such as prisons, boarding schools, and military academies (e.g., Ashworth \& Walker, 1972; Kirkham, 2000). As such, our results dovetail nicely with research on sexual fluidity in women, which indicates that current social context and situational factors (e.g., peer group membership, topical socio-cultural permissiveness) affect sexual and romantic opportunities, which can, in turn, influence the expression of some women's sexual attraction to same-sex and opposite-sex partners (Diamond, 2008). The mere presence of a male versus a female sexual partner appears to influence a woman's pattern of sexual activity. As Hite (2004) demonstrates, the duration of sexual activity in lesbian couples is longer than for heterosexual couples, they exhibit a greater variety of sexual behaviors and there is a greater emphasis on general body contact as opposed to the more specific focus in heterosexual couples on genital contact.

\section{A Female Mounting Culture in Japanese Macaques?}

Our study suggests that FMM and FFM may be cultural sexual practices in the Arashiyama-E group of Japanese macaques. In ethological parlance, a traditional (or cultural) behavior is typically defined as (1) a geographically variable behavioral practice, (2) possibly locally adaptive, (3) persistent in several individual over generations (or at least a number of years), and (4) dependent on social influences for its expression, diffusion or maintenance within the group (Fragaszy \& Perry, 2003).

We argue that female mounting in Japanese macaques meet, at least partly, these four criteria, even though we acknowledge that additional quantitative data would help further support our claim. First, we found substantial inter-group variation in the frequency of occurrence of FMM and FFM, i.e., being customary or habitual in some groups and rare or even absent in others. Second, although FFM in Japanese macaques does not seem to be adaptive, this sexual behavior may be the functionless by-product of an adaptation, namely FMM (Vasey, 2007). As we suggested here, FMM at Arashiyama may be an adaptation that females employ to prompt sexually disinterested or sluggish senescent males to copulate with them (see also Vasey, 2002). Once females evolved the capacity to mount and the capacity to derive sexual gratification during mounts through genital stimulation, they could do so just as easily by mounting females as mounting males (Vasey, 2007). Third, FMM and FFM have been continuously performed by several group members and across generations at Arashiyama since at least the early 1970s (Y. Takahata and M.A. Huffman, personal communications). Fourth, our inter-group comparative data suggest that the expression and/or maintenance of female mounting behavior in this species may be contingent on group-specific social conditions (i.e., relatively low levels of sex-related aggressiveness) in 
which such non-conceptive sexual mounts are tolerated. As pointed out by Fragaszy \& Perry (2003), social influence in the diffusion of cultural behaviors should be considered "in its broadest meaning," and encompass "all the ways that animals can develop shared behaviors that depend in some way upon the social context for their repeated generation" (p. 8). Even though this study does not provide direct evidence for the social transmission of female mounting behavior within a group of Japanese macaques, we argue that relatively high levels of social tolerance towards FMM and FFM (associated with the presence of "mellow males") are likely conducive to the expression and maintenance of these two nonconceptive sexual behaviors. Overall, this pattern suggests the existence of a female mounting culture, socially maintained in the Arashiyama-E group of Japanese macaques.

Our data showed similarities in FMM and FFM frequency in three pairs of neighboring groups located at geographically isolated field sites (Minoo, Shodoshima, and Takasakiyama; Figure 1). Albeit preliminary, this pattern is suggestive of the notion of "cultural zones." Cultural zones are areas where different groups share space and behavioral practices (Leca et al., 2007). With regards to the behaviors under study, cultural zones may arise when two neighboring groups come into occasional contact during the mating season and inter-group observational learning of female mounting patterns occurs. Even though alternative explanations should be considered (e.g., intra-population genetic variation, groupspecific demographic settings), we argue that social influences (thus, cultural factors) may account for some inter-group covariation in the frequency and structural diversity of FMM and FFM. To further examine the possibility of cultural zones for female mounting behaviors in Japanese macaques, we urge future studies to collect relevant data from additional free-ranging neighboring groups, or to experimentally provide opportunities for inter-group observational learning, between a group where these behaviors are absent and a group where they are customary, under the controlled conditions of captive settings.

In addition, previous developmental research suggested that the routine occurrence of homosexual consortships between adult females in the Arashiyama-E group, with conspicuous displays of adult female-female courtship behaviors and a variety of adult FFM postures, could attract the interest of sexually motivated adolescent females (that do not typically attract adult male mates) and promote the early expression of female homosexual behavior, via a social facilitation process (Leca et al., in review). It would be difficult to explain the high prevalence of a group-specific sexual behavioral variant, such as the sitting mounting posture by females at Arashiyama (Figure 3 and 4) without invoking social transmission processes. The same could be said for the tendency of female and male mountees at Arashiyama to solicit female mounts, a behavioral pattern that is absent at the other three sites studied. Likewise, a similar argument could be made for grasping behavior, which is absent Jigokudani, but present at the two other sites studied. To some extent, the increased diversity and complexity of female mounting patterns at Arashiyama (compared to Minoo) is suggestive of a generational ratchet-like effect. According to the cumulative cultural evolution theory, the "ratchet effect" is a process in which a beneficial behavioral variant is retained until it can be improved upon, and results in behaviors or artefacts with cultural histories, i.e., that no individual could invent on their own (Tomasello, Kruger, \& Ratner, 1993). Based on the developmental trajectory of the different female mounting postures in the Arashiyama-E group (Leca et al., in press), we believe that sitting mounts are the developmental outcome of a step-by-step elaboration on earlier and simpler mounting variants (e.g., reclining mounts) and may reflect accumulated modifications of socially transmitted behavioral patterns. Indeed, by performing sitting mounts while rubbing their vulvar region against the lower back of their male or female mates, females have improved control over the amount of immediate sexual reward they obtain (Vasey \& Duckworth, 2006, 2008).

For adaptive reasons, observational learning could also account for the frequent occurrence of FMM at Arashiyama. After watching female group members preventing male consort partners from moving away by mounting them, a naïve female might be inclined to use the same tactic in order to produce the same effect. Finally, our results showed that the routine occurrence of FMM and FFM at Arashiyama was associated with relatively low levels of male aggressiveness and high levels of permissiveness. Overall, we provided several lines of evidence that indicate how social factors may affect 
the expression or maintenance of female mounting in Japanese macaques. This is a necessary condition for a cultural interpretation of the intraspecific variation that we observed ( $c f$. Fragaszy \& Perry, 2003).

With regards to the evolutionary history of female mounting at the group level, at least two scenarios are possible. First, our inter-group comparative data suggest that in some populations like Arashiyama, female Japanese macaques exhibit a high predisposition for mounting their sexual partners, particularly FFM during adolescence and young adulthood and FMM during full adulthood and senescence (Figure 1; Table 5). However, in most populations, all the aforementioned favorable sociodemographic conditions are not met, and although female mounting may occasionally be expressed by several group members, it does not reach the group-level tradition status (e.g., Minoo-F and Minoo-L groups). In theory, this scenario could be tested by experimentally removing young and middle-aged adult males in one of the two Minoo groups (in order to induce an Arashiyama-like female-biased sex ratio) and providing a systematic comparison of the frequency, prevalence, and form of FMM and FFM in these two groups over the next generations ( $c f$. Sapolsky \& Share, 2004; Vasey \& Gauthier, 2000). Second, although FMM and FFM are probably evolutionary linked, they may appear independently in some groups as idiosyncratic behavioral innovations, only performed by one or a very few female members. The occurrence of group member "specialists" in particular behavioral patterns emphasizes the role of individuality in group-level phenomena in primates. However, it has been suggested that the occasional and restricted practice of a given behavioral pattern by very few group members may not be sufficient for its long-term maintenance in the group (Leca et al., 2010). When the only practitioner of a particular behavior dies, this behavior is also bound to disappear at the group level. This scenario may account for the rare occurrence of FMM and/or FFM in some groups of Japanese macaques (Figure 1).

Our cultural interpretation of female mounting in Japanese macaques is consistent with evidence of the social transmission of courtship behaviors and mating preferences in numerous fish, bird and mammal taxa, including nonhuman primates (e.g., Freeberg, 2004; Nishida, 1980; Rees, 2004; Warner, 1990; White, King, \& West, 2002). Likewise, there are cultural explanations for the absence of female homosexual practices in some human small-scale cultures, such as the Aka foragers and Ngandu farmers of central Africa (Hewlett \& Hewlett, 2010).

Even though our results are congruent with the notion of female mounting culture in Japanese macaques, are there alternative plausible explanations for the inter-group differences in FMM and FMM reported here? According to the "method of elimination", when obvious ecological and genetic causations can be ruled out, or at least when the effects of ecological and genetic factors are likely to be minimal, then geographic variation in behavior can be regarded as mainly cultural (van Schaik, 2003). Possible effects of ecology or genetics were not addressed in this study, and therefore, they cannot be excluded. It is acknowledged that local ecological conditions (e.g., food availability and distribution) can impact reproductive mating strategies in Japanese macaques, either directly by altering ovarian cycle phases in females, or indirectly by shaping group size and composition, which, in turn, affect heterosexual mating competition (reviewed in Fujita, 2010). Future research should examine possible ecological influences on non-conceptive sexual patterns, such as FMM and FFM. Similarly, genetic factors cannot be eliminated. Indeed, the three populations in which FMM and FFM have been reported to occur customarily, or at least habitually, fall within a single haplogroup (A1), which is exclusive to central and western Honshu, Japan (Vasey \& Jiskoot, 2010). These three populations originate from: (1) Arashiyama, Kyoto Prefecture (Arashiyama-E and its two sister groups translocated outside Japan, namely the semi-free ranging Arashiyama-West group, USA: Gouzoules \& Goy, 1983, and the captive Montréal group, Canada: Vasey, 2002), (2) Minoo, Osaka Prefecture (Minoo-F and Minoo-L groups), and (3) Wakasa, Tottori Prefecture (captive Wakasa-B group, Primate Research Institute, Inuyama; Vasey \& Reinhart, 2009; Gunst, unpublished data).

It is noteworthy that the use of the method of elimination has been severely criticized, for two main reasons: (1) ruling out all possible ecological and genetic correlates of intraspecific behavioral variation is practically impossible and logically inadequate (like proving the null hypothesis), and (2) any inter-group behavioral differences may rarely be due to culture alone, but may often be attributed to a complex interaction between ecological conditions, genetic components, and cultural influences 
(Fragaszy \& Perry, 2003). Therefore, genetic, ecological, and cultural processes could all be responsible for intraspecific variation in female mounting in Japanese macaques. However, we suggest that arbitrary behavioral patterns such as female mount postures, solicitations of female mounting, and female-to-male inter-mount grasping behavior, could be purely cultural, as any alternative explanation is difficult to imagine.

\section{Acknowledgements}

This study was funded by the following agencies: Natural Sciences and Engineering Research Council of Canada (NSERC), Alberta Innovates Health Solutions (AIHS), Japan Society for the Promotion of Science (JSPS), American Institute of Bisexuality (AIB), the L. S. B. Leakey Foundation, as well as the Office of the Dean of Arts and Science and the Office of Research Services at the University of Lethbridge, Alberta, Canada. We thank Penny D'Agnone for help securing AIHS funding. We are grateful to the researchers and staff for kindly providing valuable information from their respective field research sites: S. Hayakawa, M. A. Huffman, F. Kanchi, A. J. J. MacIntosh, N. Nakagawa, Y. Tsuji, K. Watanabe, and K. Yamada (Kyoto University), M. Shimada (Teikyo University of Science and Technology), Y. Takahata (Kwansei Gakuin University), S. Turner (McGill University), P. Zhang (Sun Yat-sen University), H. Kurita (Board of Education, Oita City), S. Asaba (Arashiyama Monkey Park), Y. Iwanaga (Minoo Field Station), T. Shimomura (Takasakiyama Natural Zoo), T. Kikuma (Miyajima Ropeway Company), as well as H. Takefushi and T. Hagiwara (Jigokudani Monkey Park). We thank J. and N. Pontecorvo (Pontecorvo Productions) for additional information on the monkeys of Jigokudani Monkey Park. We thank J. Vonk and three anonymous reviewers for constructive comments on a previous version of the manuscript.

\section{References}

Alberts, S. C. (2012). Magnitude and sources of variation in male reproductive performance. In J. C. Mitani, J. Call, P. M. Kappeler, R. A. Palombit, \& J. B. Silk (Eds.), The evolution of primate societies (pp. 412-431). Chicago: The University of Chicago Press.

Altmann, J. (1974). Observational study of behavior: Sampling methods. Behaviour, 49, 227-267.

Ashworth, A. E., \& Walker, W. M. (1972). Social structure and homosexuality: A theoretical appraisal. The British Journal of Sociology, 23, 146-158.

Beach, F. A. (1968). Factors involved in the control of mounting behavior by female mammals. In M. Diamond (Ed.), Perspectives in reproduction and sexual behavior: A memorial to William C. Young (pp. 83-1 31). Bloomington, IN: Indiana University Press

Dagg, A. I. (1984). Homosexual behaviour and female-male mounting in mammals - a first survey. Mammal Review, 14, 155-185.

Diamond, L. M. (2008). Sexual fluidity: Understanding women's love and desire. Cambridge, MA: Harvard University Press.

Dixson, A. F. (2012). Primate sexuality: Comparative studies of the prosimians, monkeys, apes, and humans. Oxford, UK: Oxford University Press.

Dobzhansky, T. (1937). Genetics and the origins of species. New York: Columbia University Press.

Fragaszy, D. M. \& Perry, S. (2003). Towards a biology of traditions. In D. M. Fragaszy \& S. Perry (Eds.), The biology of traditions: Models and evidence (pp. 1-32). Cambridge, UK: Cambridge University Press.

Freeberg, T. M. (2004). Social transmission of courtship behavior and mating preferences in brown-headed cowbirds, Molothrus ater. Learning \& Behavior, 32, 122-130.

Fujita, S. (2010). Interaction between male and female mating strategies and factors affecting reproductive outcome. In N. Nakagawa, M. Nakamichi, \& H. Sugiura (Eds.), The Japanese macaques (pp. 221-239). Tokyo: Springer.

Goldizen, A. W. (1988). Tamarin and marmoset mating systems: Unusual flexibility. Trends in Ecology and Evolution, 3, 36-40.

Goodall, J. (1986). The chimpanzees of Gombe: Patterns of behavior. Cambridge, MA: Harvard University Press.

Gouzoules, H., \& Goy, R. W. (1983). Physiological and social influences on mounting behaviour of troop living female monkeys (Macaca fuscata). American Journal of Primatology, 5, 39-49. 
Green. S. (1975). Dialects in Japanese monkeys: Vocal learning and cultural transmission of locale-specific vocal behavior? Zeitschrift für Tierpsychologie, 38, 304-314.

Gunst, N., Leca, J.-B., \& Vasey, P. L. (In review). Influence of sexual competition and social context on homosexual behaviour in adolescent female Japanese macaques. American Journal of Primatology.

Gunst, N., Leca, J.-B., \& Vasey, P. L. (2013). Development of sexual and socio-sexual behaviors in free-ranging juvenile male Japanese macaques, Macaca fuscata. Behaviour, 150, 1225-1254.

Hite, S. (2004). The Hite report: A national study of female sexuality. New York: Seven Stories Press

Hewlett, B. S., \& Hewlett, B. L. (2010). Sex and searching for children among Aka foragers and Ngandu farmers of Central Africa. African Study Monographs, 31, 107-125.

Itani, J., \& Nishimura, A. (1973). The study of infra-human culture in Japan. In: E. W. Menzel, Jr (Ed.), Symposia of the fouth international congress of primatology, volume 1: Precultural primate behavior (pp. 26-50). Basel, Switzerland: Karger.

Kirkham, G. L. (2000). Homosexuality in prison. In P. C. R. Rust (Ed.), Bisexuality in the United States (pp. 250267). New York: Columbia University Press.

Kvarnemo, C., \& Ahnesjö, I. (2002). Operational sex ratios and mating competition. In I. C. Hardy (Ed.), Sex ratios: Concepts and research methods (pp. 366-382). Cambridge, UK: Cambridge University Press.

Leca, J.-B., Gunst, N., \& Huffman, M. A. (2007). Japanese macaque cultures: Inter- and intra-troop behavioural variability of stone handling patterns across 10 troops. Behaviour, 144, 251-281.

Leca, J.-B., Gunst, N., \& Huffman, M. A. (2010). The first case of dental flossing by a Japanese macaque (Macaca fuscata): Implications for the determinants of behavioral innovation and the constraints on social transmission. Primates, 51, 13-22.

Leca, J.-B., Gunst, N., \& Vasey, P. L. (In press). Development of sexual behavior in free-ranging female Japanese macaques. Developmental Psychobiology.

Leca, J.-B., Gunst, N., \& Vasey, P. L. (In review). Comparative development of heterosexual and homosexual behaviors in free-ranging female Japanese macaques. Archives of Sexual Behavior.

Lorenz, K. (1970). Studies in animal and human behavior. London: Methuen.

Muller, M. N., \& Wrangham, R. W. (2009). Sexual coercion in primates and humans: An evolutionary perspective on male aggression against females. Cambridge, MA: Harvard University Press.

Nakagawa, N. (2010). Intraspecific differences in the social structure of Japanese macaques: A revival of lost legacy by updated knowledge and perspective. In N. Nakagawa, M. Nakamichi, \& H. Sugiura (Eds.), The Japanese macaques (pp. 271-290). Tokyo: Springer.

Nakagawa, N., Nakamichi, M., \& Yamada, K. (2011). Report on the questionnaire for infrequently-observed behaviors in Japanese macaques. Primate Research, 27, 111-125. (in Japanese)

Nishida, T. (1980). The leaf-clipping display: A newly-discovered expressive gesture in wild chimpanzees. Journal of Human Evolution, 9, 117-128.

O’Neill, A. C., Fedigan, L. M., \& Ziegler, T. E. (2006). Hormonal correlates of post-conceptive mating in female Japanese macaques. Laboratory Primate Newsletter, 45, 1-4.

Paoli, T. (2009). The absence of sexual coercion in bonobos. In M. N. Muller \& R. W. Wrangham (Eds.), Sexual coercion in primates and humans: An evolutionary perspective on male aggression against females (pp. 410-423). Cambridge, MA: Harvard University Press.

Pavelka, M. S. M., \& Fedigan, L. M. (1999). Reproductive termination in female Japanese monkeys: A comparative life history perspective. American Journal of Physical Anthropology, 109, 455-464.

Rees, P. A. (2004). Some preliminary evidence of the social facilitation of mounting behavior in a juvenile bull Asian elephant (Elephas maximus). Journal of Applied Animal Welfare Science, 7, 49-58.

Sakura, O. (1989). Variability in contact calls between troops of Japanese macaques: A possible case of neutral evolution of animal culture. Animal Behaviour, 38, 900-902.

Sapolsky, R. M., \& Share, L. J. (2004). A pacific culture among wild baboons: Its emergence and transmission. PLoS Biology, 2, 534-541.

Siegel, S., \& Castellan, N. J. (1988). Nonparametric statistics for the behavioral sciences. Singapore: McGraw-Hill.

Silk, J. B. (2007). Social components of fitness in primate groups. Science, 317, 1347-1351.

Sitzmann, B. D., Urbanski, H. E., \& Ottinger, M. A. (2008). Aging in male primates: Reproductive decline, effects of calorie restriction and future research potential. Age, 30, 157-168.

Sprague, D. S. (1998). Age, dominance rank, natal status, and tenure among male macaques. American Journal of Physical Anthropology, 105, 511-521. 
Stephenson, G. R. (1973). Testing for group specific communication patterns in Japanese macaques. In E. W. Menzel, Jr (Ed.), Symposia of the fouth international congress of primatology, volume 1: Precultural primate behavior (pp. 51-75). Basel, Switzerland: Karger.

Tanaka, I. (1998). Social diffusion of modified louse egg-handling techniques during grooming in free-ranging Japanese macaques. Animal Behaviour, 56, 1229-1236.

Thierry, B., Iwaniuk, A. N., \& Pellis, S. M. (2000). The influence of phylogeny on the social behaviour of macaques (Primates: Cercopithecidae, genus Macaca). Ethology, 106, 713-728.

Tomasello, M., Kruger, A. C., \& Ratner, H. H. (1993). Cultural learning. Behavioural and Brain Sciences, 16, 495552.

van Schaik, C. P. (2003). Local traditions in orangutans and chimpanzees: Social learning and social tolerance. In D. M. Fragaszy \& S. Perry (Eds.), The biology of traditions: Models and evidence (pp. 297-328). Cambridge, UK: Cambridge University Press.

Vasey, P. L. (2002). Same-sex sexual partner preference in hormonally and neurologically unmanipulated animals. Annual Review of Sex Research, 13, 141-179.

Vasey, P. L. (2004). Sex differences in sexual partner acquisition, retention, and harassment during female homosexual consortships in Japanese macaques. American Journal of Primatology, 64, 397-409.

Vasey, P. L. (2007). Function and phylogeny: The evolution of same-sex sexual behaviour in primates. Journal of Psychology and Human Sexuality, 18, 215-244.

Vasey, P. L., \& Duckworth, N. (2006). Sexual reward via vulvar, perineal and anal stimulation: A proximate mechanism for female homosexual mounting in Japanese macaques. Archives of Sexual Behavior, 35, 523532.

Vasey, P. L., \& Duckworth, N. (2008). Female-male mounting in Japanese macaques: The proximate role of sexual reward. Behavioural Processes, 77, 405-407.

Vasey, P. L., Foroud, A., Duckworth, N., \& Kovacovsky, S. D. (2006). Male-female and female-female mounting in Japanese macaques: A comparative analysis of posture and movement. Archives of Sexual Behavior, 35, 117-129.

Vasey, P. L., \& Gauthier, C. (2000). Skewed sex ratios and female homosexual activity in Japanese macaques: An experimental analysis. Primates, 41, 17-25.

Vasey, P. L, \& Jiskoot, H. (2010). The biogeography and evolution of female homosexual behavior in Japanese macaques. Archives of Sexual Behavior, 39, 1439-1441.

Vasey, P. L., \& Reinhart, C. (2009). Female homosexual behavior in a new group of Japanese macaques: Evolutionary implications. Laboratory Primate Newsletter, 48, 8-10.

Vasey, P. L., \& VanderLaan, D. P. (2012). Is female homosexual behaviour in Japanese macaques truly sexual? In J.-B. Leca, M. A. Huffman, \& P. L. Vasey (Eds.), The monkeys of stormy mountain: 60 years of primatological research on the Japanese macaques of Arashiyama (pp. 153-172). Cambridge, UK: Cambridge University Press.

Verrell, P. A. (1999). Geographic variation in sexual behaviour: Sex, signals, and speciation. In S. A. Foster \& J. A. Endler (Eds.), Geographic variation in behavior: Perspective on evolutionary mechanisms (pp. 262-286). New York: Oxford University Press.

Warner, R. R. (1990). Male versus female influences on mating-site determination in a coral reef fish. Animal Behaviour, 39, 540-548.

White, D. J., King, A. P., \& West, M. J. (2002). Facultative development of courtship and communication in juvenile male cowbirds (Molothrus ater). Behavioral Ecology, 13, 487-496.

Wickler, W. (1967). Socio-sexual signals and their intra-specific imitation among primates. In D. Morris (Ed.), Primate ethology (pp. 69-79). Chicago: Aldine.

Wolfe, L. D. (1986). Sexual strategies of female Japanese macaques (Macaca fuscata). Human Evolution, 1, 267275.

Zhang, P., \& Watanabe, K. (In press). Intraspecies variation in dominance style of Macaca fuscata. Primates. 